November 2005

\title{
Posterior longitudinal ligament status in cervical spine bilateral facet dislocations
}

John A. Carrino

Harvard Medical School \& Brigham and Women's Hospital

Geoffrey L. Manton

Thomas Jefferson University Hospital

William B. Morrison

Thomas Jefferson University Hospital

Alex R. Vaccaro

Thomas Jefferson University Hospital and The Rothman Institute

Mark E. Schweitzer

New York University \& Hospital for Joint Diseases

Follow this and additional works at: https://jdc.jefferson.edu/orthofp

Part of the Orthopedics Commons

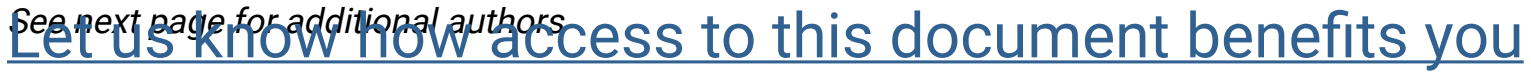

\section{Recommended Citation}

Carrino, John A.; Manton, Geoffrey L.; Morrison, William B.; Vaccaro, Alex R.; Schweitzer, Mark E.; and Flanders, Adam E., "Posterior longitudinal ligament status in cervical spine bilateral facet dislocations" (2005). Department of Orthopaedic Surgery Faculty Papers. Paper 3.

https://jdc.jefferson.edu/orthofp/3

This Article is brought to you for free and open access by the Jefferson Digital Commons. The Jefferson Digital Commons is a service of Thomas Jefferson University's Center for Teaching and Learning (CTL). The Commons is a showcase for Jefferson books and journals, peer-reviewed scholarly publications, unique historical collections from the University archives, and teaching tools. The Jefferson Digital Commons allows researchers and interested readers anywhere in the world to learn about and keep up to date with Jefferson scholarship. This article has been accepted for inclusion in Department of Orthopaedic Surgery Faculty Papers by an authorized administrator of the Jefferson Digital Commons. For more information, please contact: JeffersonDigitalCommons@jefferson.edu. 


\section{Authors}

John A. Carrino, Geoffrey L. Manton, William B. Morrison, Alex R. Vaccaro, Mark E. Schweitzer, and Adam E. Flanders 


\title{
Posterior longitudinal ligament status in cervical spine bilateral facet dislocations
}

\author{
John A. Carrino \\ William B. Morrison \\ Geoffrey L. Manton \\ Alex R. Vaccaro \\ Mark E. Schweitzer \\ Adam Flanders
}

\author{
J. A. Carrino (corresponding author) \\ Harvard Medical School \\ Department of Radiology \\ Brigham and Women's Hospital \\ 75 Francis St. \\ Boston, MA 02115, USA \\ e-mail: jcarrino@partners.org \\ Tel.: +1-617-7327537 \\ Fax: +1-617-2786976 \\ G. L. Manton, W. B. Morrison \& A. E. Flanders \\ Department of Radiology \\ Thomas Jefferson University Hospital \\ Philadelphia, PA, USA
}

\author{
A. R. Vaccaro \\ Department of Orthopedic Surgery \\ The Rothman Institute \\ Thomas Jefferson University Hospital \\ Philadelphia, PA, USA \\ M. E. Schweitzer \\ Department of Radiology \\ New York University \\ Hospital for Joint Diseases \\ New York, NY, USA
}

\section{Abstract}

Objective: It is generally accepted that cervical spine bilateral facet dislocation results in complete disruption of the posterior longitudinal ligament. The goal of this study was to evaluate the integrity of numerous spine-stabilizing structures by MRI, and to determine if any associations between injury patterns exist with respect to the posterior longitudinal ligament status.

Design: Retrospective case series.

Patients: A retrospective review was performed of 30 cervical spine injury subjects with bilateral facet dislocation. Assessment of 1.5T MRI images was carried out for: intervertebral disc disruption, facet fracture, and ligamentous disruption. Statistical analyses were performed to evaluate for associations between various injury patterns and posterior longitudinal ligament status.

Results: The frequency of MRI abnormalities was: anterior longitudinal ligament disruption (26.7\%), disc herniation or disruption (90\%), posterior longitudinal ligament disruption (40\%), facet fracture (63.3\%) and disruption of the posterior column ligament complex (97\%). There were no significant associations between injury to the posterior longitudinal ligament and other structures. Compared to surgical reports, MRI was accurate for determining the status for 24 of 26 ligaments (three of three anterior longitudinal ligament, seven of nine posterior longitudinal ligament, and 14 of 14 posterior column ligament complex) but generated false negatives in two instances (in both MRI showed an intact posterior longitudinal ligament that was torn at surgery). 
Conclusions: In contradistinction to the existing concept, the posterior longitudinal ligament can remain intact in a substantial proportion of hyperflexion injuries that produce bilateral cervical facet dislocation. Posterior longitudinal ligament integrity is not associated with any other injury pattern related to the anterior longitudinal ligament, intervertebral disc or facet fracture.

\section{Introduction}

Bilateral facet dislocation is a relatively rare, but clinically significant cervical spine injury. Classically considered the result of a pure hyperflexion injury, bilateral facet dislocation is defined as the anterior displacement of both facet joint articular facets at the same level, such that posterior-inferior margins of the inferior articular processes lie at least atop the superior articular processes of the subjacent vertebra (incomplete, or "perched facets"), or fully beyond said processes, so that the dislocated articular masses sit in the inferior portion of the underlying intervertebral foramina (complete, frank, or "jumped facets”)[1]. The vertebral body at the dislocated level in anteriorly displaced, and may reside upwards of $50 \%$ beyond the anteroposterior vertebral body dimension in complete bilateral facet dislocation.

Because of the forces involved and degree of distraction, bilateral facet dislocation also results in extensive soft-tissue injury, causing an unstable cervical spine. Complete disruption of the posterior column ligament complex, the posterior longitudinal ligament, the intervertebral disc, and the anterior longitudinal ligament have all been described [2, 3]. In addition to the previously mentioned injuries, fractures of the articular pillars may be present in bilateral facet dislocation, although these have been thought to be clinically less relevant in comparison to the ligamentous injuries [4]. Bilateral facet fracture-dislocation has traditionally been considered a variation of bilateral facet dislocation, and often has less severe anterolisthsis.

MRI has become a valuable tool in assessing the soft tissues of the cervical spine, especially in trauma situations. While extensive research has been performed in various aspects of MRI of the cervical spine, very little research has examined the MRI appearance of the soft-tissue injuries typically involved with bilateral facet dislocation [5]. Therefore, our goal was to use MRI to evaluate the integrity of the soft tissues typically injured in bilateral facet dislocation. The purpose of this investigation was to determine the frequency of an intact posterior longitudinal ligament in the setting of bilateral facet dislocation, as well as to determine associations with other injury patterns of the anterior longitudinal ligament, intervertebral disc or facet fracture.

\section{Materials and methods}

This study was approved by the institutional IRB (internal review board) as a medical records review, and therefore informed consent was not obtained from the research subjects. The study sample was selected by a radiology information system search of reports on patients admitted to our Level 1 regional spinal cord injury center from January 1997 to March 2002 with the diagnosis of bilateral facet dislocation made either by radiography or computed tomography, who underwent subsequent cervical spine MRI. The study group sample was comprised of 30 subjects whose demographics are shown in Table 1 . The neurological status of the subjects ranged from fully intact to complete tetraplegia (ASIA grade A). 
Table 1 Subject characteristics

$N=30$

\begin{tabular}{ll}
\hline Gender & $\begin{array}{l}\text { Male=26 } \\
\text { Female=4 }\end{array}$ \\
Age (in years) & $\begin{array}{l}\text { Mean=39.8 } \\
\text { Range 20-85 }\end{array}$ \\
$\begin{array}{l}\text { Mechanism of } \\
\text { injury }\end{array}$ & $\begin{array}{l}\text { Motor vehicle-/motorcycle-related accident=19 } \\
\text { Sports-related injury=4 } \\
\text { Fall=7 }\end{array}$ \\
\hline
\end{tabular}

On all subjects, cervical spine MRI was performed after closed traction reduction (or attempted reduction) of the facet dislocation. All MRI examinations were performed using a 1.5T scanner (Signa; General Electric, Milwaukee, WI, USA) with a combination of sagittal and axial images. All sagittal images were acquired with 22-cm field of view, 256×224 matrix, and 4-mm slice thickness. The sagittal pulse sequences were: T1-weighted spin echo (TE=8 ms, $\mathrm{TR}=350 \mathrm{~ms}$ ) or proton density (intermediate weighted) fast spin echo ( $\mathrm{TE}=15 \mathrm{~ms}, \mathrm{TR}=2800 \mathrm{~ms}$ ) and T2-weighted sagittal fast spin echo (TE=90 ms, $\mathrm{TR}=2800 \mathrm{~ms}$ ) with fat suppression. All axial images were acquired with 20 -cm field of view, 256×256 matrix. The axial pulse sequences were: $\mathrm{T} 2$ ( $\mathrm{TE}=80 \mathrm{~ms}$, $\mathrm{TR}=3817 \mathrm{~ms}, 4-\mathrm{mm}$ slice thickness) and 3D gradient echo (TE=14 ms, TR=600 ms, flip angle=20 degrees, 2.8-mm slice thickness).

Two radiologists experienced in spine imaging (AEF, JAC) performed a retrospective consensus review for each case using a standardized score sheet, assessing for the following criteria: disruption of the anterior longitudinal ligament, posterior longitudinal ligament, posterior column ligament complex, and intervertebral disc. Acute disc disruption was defined as loss of continuity of the anulus fibrosis, typically manifested by abnormal fluid signal intensity on T2-weighted sequences in the outer portion of the disc. The posterior column ligament complex is comprised of the supraspinous and interspinous ligaments as well as the ligamentum flavum. Evaluation of the complex was made along the midline sagittal sections, and was characterized as intact or disrupted. Disruption of the anterior longitudinal ligament, posterior longitudinal ligament, and posterior column ligament complex was defined as discontinuity of these structures, or abnormal fluid signal intensity within these structures on T2-weighted sequences without an underlying normal expected architecture. Because of artifacts secondary to motion, technique, or contrast, the longitudinal ligaments were classified as intact if they were seen as contiguous on any sequence. Additionally, the classification of intact but elevated was made for the anterior longitudinal ligament and posterior longitudinal ligament in cases where the fibers of these ligaments were not disrupted, but in which the location of the ligament was displaced from normal secondary to an abnormality in the juxtaposed tissues, mainly from anterolithesis (Fig. 1). 


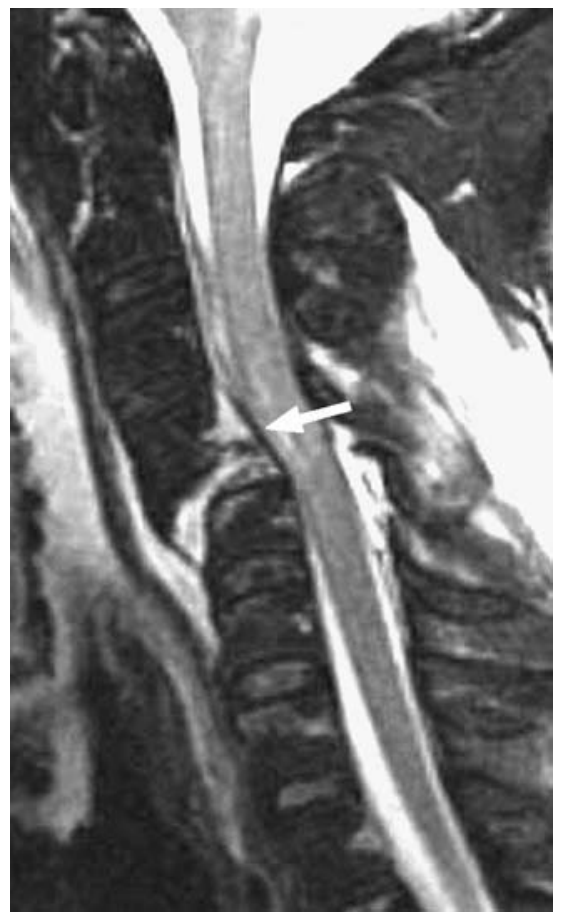

Fig. 1 True-negative intact posterior longitudinal ligament by MRI. 29-year-old male with bilateral interfacetal dislocation at C4-5. Sagittal T2-weighted fast spin echo fat-suppressed image shows an intact but elevated posterior longitudinal ligament, manifested by a continuous linear low signal intensity structure (arrow) extending from the posterosuperior aspect of C4 to the posterosuperior aspect of $\mathrm{C} 5$, that was confirmed at surgery.

\section{Statistical analyses}

Descriptive analyses were performed on the frequency of lesions based on the MRI interpretation. The sample was dichotomized into two groups (posterior longitudinal ligament intact and posterior longitudinal ligament disrupted). Assessments were made to determine if there were any associations with the other dichotomized findings noted at imaging (anterior longitudinal ligament, facet fracture and intervertebral disc status), excluding posterior ligament complex disruption, since this was present in virtually all cases. Statistical analysis was accomplished by construction of two-by-two contingency tables, performing pair-wise comparisons with each of the other findings. The level of statistical significance was determined using the Fisher exact test. A p-value of 0.05 was considered significant. Comparisons with surgical reports were available for a subset of subjects $(n=14)$. Surgical confirmation of ligamentous disruption was not available for every case, due to a variety of factors including 1) no surgery, 2) limited visualization secondary to surgical approach, and 3) lack of documentation in the operative note. 


\section{Results}

Cervical-spine MRI was performed after closed-traction reduction in 43.3\% (13/30) of cases, and in $56.7 \%(16 / 30)$ after attempted but unsuccessful reduction of the facet dislocation. The frequencies of MRI findings are summarized in Table 2. Of the 30 subjects studied by MRI, 22 (73.3\%) had an intact anterior longitudinal ligament (15/22 were intact but elevated) and 8 (26.7\%) were disrupted. The posterior longitudinal ligament was intact (Fig. 1)in 18 subjects (60.0\%)(16/18 were intact but elevated), and 12 (40.0\%) showed disruption (Fig. 2). Only three subjects (10.0\%) demonstrated an intact intervertebral disc, and 27 (90\%) were acutely disrupted. Facet fracture was noted in 19 of subjects (63.3\%). No statistically significant associations were found between posterior longitudinal ligament status and anterior longitudinal ligament $(\mathrm{p}=0.465)$, facet fracture $(p=0.503)$ and intervertebral disc status $(p=0.518)$.

Table 2 Frequency distribution of lesions by MRI findings

\begin{tabular}{lcc} 
Structure & Intact & Disrupted \\
\hline Intervertebral facets & $36.7 \%(11 / 30)$ & $63.3 \%(19 / 30)$ \\
Intervertebral disc & $10.0 \%(3 / 30)$ & $90.0 \%(27 / 30)$ \\
Longitudinal ligament & & \\
$\quad$ Anterior & $73.3 \%(22 / 30)$ & $26.7 \%(8 / 30)$ \\
$\quad$ Posterior & $60.0 \%(18 / 30)$ & $40.0 \%(12 / 30)$ \\
Posterior column ligament complex & & \\
$\quad$ Interspinous ligament & $3.3 \%(1 / 30)$ & $96.7 \%(29 / 30)$ \\
$\quad$ Supraspinous ligament & $3.3 \%(1 / 30)$ & $96.7 \%(29 / 30)$ \\
$\quad$ Ligamentum flavum & $10.0 \%(3 / 30)$ & $90.0 \%(27 / 30)$
\end{tabular}

Surgical reports were available on 14 of the 30 cases (46.7\%). However, not all ligaments were commented on in every operative note. There was surgical concordance of two intact and one torn anterior longitudinal ligaments, five intact and two disrupted posterior longitudinal ligaments, and 14 disrupted posterior column ligament complexes. There was some discordance with two posterior longitudinal ligaments that were interpreted as intact on MRI but considered disrupted at surgical assessment (Fig. 3).

\section{Discussion}

This was a retrospective case series of 30 subjects with bilateral facet dislocations who underwent MRI. Contrary to current concepts, we found that the posterior longitudinal ligament does not uniformly tear in every instance of this lesion.

Bilateral facet dislocation is a serious cervical-spine injury that often results in marked spinal-cord injury, notably tetraplegia. Given the instability of this injury, neurological deficits can manifest as well as worsen sometime after the onset of injury if not treated appropriately. Using cadaveric experimental models, the classic article by Beatson [2] in 1963 described the soft-tissue injuries involved with bilateral facet dislocation that causes this spinal instability. In this model, bilateral facet dislocation could not occur unless the superior vertebral body slipped forward at least 50\% of its anteroposterior length. For 


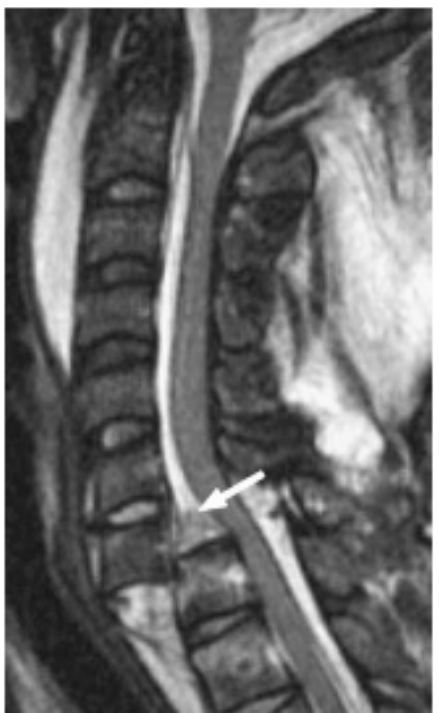

Fig. 2 True-positive tear posterior longitudinal ligament by MRI. 36-year-old male with bilateral interfacetal dislocation at C7-T1. Sagittal T2-weighted fast spin echo fat-suppressed image shows disruption of the posterior longitudinal ligament, manifested by absence of a linear low signal intensity structure (arrow) in its expected location, that was confirmed at surgery.

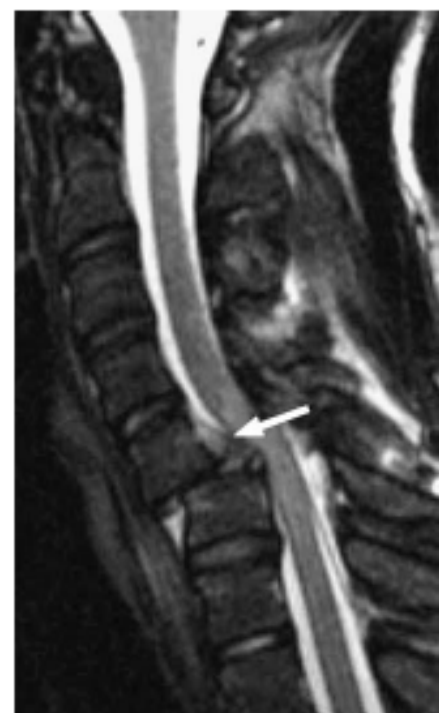

Fig. 3 False-negative intact posterior longitudinal ligament by MRI. 41-year-old male with bilateral interfacetal dislocation at C5-6. Sagittal T2-weighted fast spin echo fat-suppressed image shows an intact posterior longitudinal ligament manifested by a linear low signal intensity structure (arrow). However, the posterior longitudinal ligament was torn at surgery, making this a falsenegative MRI.

this to occur, the facet capsules, interfacetal ligaments, interspinous ligament, anulus fibrosis, and the posterior longitudinal ligament needed to be ruptured. There are no clinical studies which confirm this pattern of injury outside of the cadaveric model.

MRI can provide new insight into cervical-spine flexion-distraction injuries as a pathoanatomical surrogate tool. Given the superior visualization of soft tissues, MRI has been well-accepted as a useful imaging modality in spinal trauma [6-12], and can be used to assist in guiding clinical management [6,9]. An early case report from 1993 describes the utility of MRI in diagnosing soft-tissue injuries in bilateral facet dislocation, namely intervertebral disc and posterior longitudinal ligament rupture [8]. In this report, however, one case of bilateral facet dislocation was noted with an intact posterior longitudinal ligament on MRI. Similarly, another study [13] which used MRI to examine nine subjects with bilateral facet dislocation noted an intact anterior longitudinal ligament in two, and an intact posterior longitudinal ligament in three of the subjects. This investigation was an MRI study without correlation to surgical findings. Another study by Vaccaro, Madigan, Schweitzer et al. [5] retrospectively analyzed 48 subjects with flexion-distraction injuries. In cases of bilateral facet dislocation $(n=23)$, they found disruption of: the anterior longitudinal ligament in $65.2 \%$, the posterior longitudinal ligament in $56.5 \%$, and the posterior column ligament complex in $68.2 \%$. Our results were lower for disruption of the anterior longitudinal ligament (26.7\%) and posterior longitudinal ligament (33.3\%) and higher for the posterior column ligament complex (96.7\%). This could be partially explained by the fact that the Vaccaro investigators classified some cases as "indeterminate" (approximately 4\% of their anterior longitudinal ligament and $4 \%$ of their posterior longitudinal ligament cases), which was not done in our study. In addition, the criteria used to define ligamentous integrity in our study were more stringent than those used by prior investigators. Prior studies classified complete ligamentous disruption when only a part of the ligament appeared damaged on MRI. 
Our findings, of only $26.7 \%$ complete disruption of the anterior longitudinal ligament and $40 \%$ complete disruption of the posterior longitudinal ligament in cases of bilateral facet dislocation, are contrary to expected results, given the classic literature [2, 3, 14]. Beatson describes the injuries involved with complete bilateral facet dislocation, and suggests less severe soft-tissue injury occurs only with incomplete bilateral facet dislocation. One possible explanation is that the degree of elasticity of the ligaments and other soft tissues in cadavers may differ from the in-vivo state, and therefore a cadaveric model does not necessarily predict clinical results. However, even the surgically proved cases of an intact posterior longitudinal ligament in our series merely document the presence of visibly intact fibers, and not the functional capability or mechanical stability. Nonetheless, our data show that complete anatomic disruption of the anterior longitudinal ligament and posterior longitudinal ligament is not a prerequisite to developing a bilateral facet dislocation.

There are several limitations to this retrospective case series. Lack of surgical correlation of the ligament status data on all subjects was the main limitation of our study. Another limitation was the fact that some imaging was performed post-reduction (or attempted post-reduction), which allowed for variability in injuries from onset of injury to time of imaging, as well as an inability to define the exact degree of posttraumatic subluxation. Also, the longitudinal ligaments have a variable appearance in the dislocated versus reduced state. Hence the designation of intact but elevated, which was present in pre-operative non-reducible injuries related to anterolisthesis. We acknowledge that there is very probably altered mechanical integrity and resultant stability of these ligaments. Therefore, even though the intact but elevated ligaments are morphologically intact, they may be mechanically deficient through stretching or partial tearing. Therefore, assessment of a ligament through simple observation of continuity on MRI may be problematic -- morphologic integrity may not represent mechanical integrity -- but nonetheless these data suggest that complete posterior longitudinal ligament morphologic disruption is not a mandatory prerequisite to bilateral interfacetal dislocation, and is not an unusual finding at MRI. Prospective studies with surgical confirmation, using both direct visual inspections and perhaps a biomechanical assessment of ligamentous integrity, would be helpful to further confirm our findings, but may be problematic to accomplish given the nature of the injury and the surgical exposure.

In summary, the posterior longitudinal ligament can remain intact in bilateral facet dislocation, contrary to the classical literature based on cadaveric models. An intact ligament is not associated with any other particular injury pattern. The presence of this MRI finding probably warrants re-evaluation of the current clinical concepts regarding this type of cervical-spine injury.

\section{References}

1. Harris JH, Mirvis SE. The radiology of acute cervical spine trauma, 3rd edn. Williams and Wilkins, Philadelphia 1996

2. Beatson TR. Fractures and dislocations of the cervical spine. J Bone Joint Surg Br 1963;45:21-35

3. Dosch JC. Trauma: conventional radiological study in spine injury. Springer, Berlin Heidelberg New York 1985

4. Bedbrook GM. Stablility of spinal fractures and fracture dislocations. Paraplegia 1971;9:23-32

5. Vaccaro AR, Madigan L, Schweitzer ME, et al. Magnetic resonance imaging analysis of soft tissue disruption after flexion-distraction injuries of the sub-axial cervical spine. Spine 2001;26 (17):1866-1872

6. Brightman RP, Miller CA, Rea GL, et al. Magnetic resonance imaging of trauma to the thoracic and lumbar spine. Spine 1992;17:541-545 
7. Flanders AE, Tartaglino LM, Friedman DP, et al. Magnetic resonance imaging in acute spinal injury. Semin Roentgenol 1992;27:271-298

8. Hall AJ, Wagle VG, Raycroft J, et al. Magnetic resonance imaging in cervical spine trauma. J Trauma 1993;34:21-26

9. Keiper MD, Zimmerman RA, Bilaniuk LT. MRI in the assessment of the supportive soft tissues of the cervical spine in acute trauma in children. Neuroradiology 1998;40:359-363

10. Rizzolo SJ, Vaccaro AR, Cotler JM. Cervical spine trauma. Spine 1994;19:2288-2298

11. Silberstein M, Tress BM, Hennessy O. Prevertebral swelling in the cervical spine injury: identification of ligament injury with magnetic resonance imaging. Clin Radiol 1992;46:318-323

12. Tehranzadeh J, Kerr R, Amster J. Magnetic resonance imaging of tendon and ligament abnormalities: Part I. spine and upper extremity. Skeletal Radiol 1992;21:1-9

13. Vaccaro AR, Falatyn SP, Flanders AE, et al. Magnetic resonance evaluation of the intervertebral disc, spinal ligaments, and spinal cord before and afterclose traction reduction of the cervical spine dislocations. Spine 1999;24:1210-1217

14. Whitley JE, Forsyth HF. The classification of cervical spine injuries. Am J Roentgenol 1960;83:633-644 